'Departamento de Ciencias Neurológicas Oriente, Facultad de Medicina, Universidad de Chile. Santiago, Chile. ${ }^{2}$ Servicio de Neurología, Hospital de Salvador, Santiago, Chile. IInstituto de Neurocirugía Dr. Asenjo, Santiago, Chile. ${ }^{4}$ Escuela de Postgrado, Universidad de Santiago de Chile. Santiago Chile.

${ }^{5}$ Biomedical Neuroscience Institute Facultad de Medicina, Universidad de Chile, Santiago, Chile. ${ }^{6}$ Program of Cellular and Molecular Biology, Center for Molecula Studies of the Cell, ICBM, Facultad de Medicina, Universidad de Chile. Santiago, Chile. ${ }^{7}$ FONDAP Center for Geroscience, Brain Health and Metabolism Santiago, Chile. aBioquímico, $\mathrm{PhD}$.

${ }^{b}$ Ingeniero en Biotecnología Molecular, PhD.

Financiamiento: Trabajo financiado por: Millennium Institute Grant P09 015-F, FONDAP-15150012 (CH) FONDECYT 1150579 (D. B. M.)

Recibido el 17 de noviembre de 2015, aceptado el 23 de marzo de 2016

Correspondencia a: Marcos Ramírez marcosirp@hotmail.com

\section{Desafíos en el diagnóstico de enfermedad de Creutzfeldt-Jakob. Caso clínico}

\author{
MARCOS RAMÍREZ ${ }^{1,3}$, ANDRÉS GALLARDO $^{1}$, \\ AARÓN VIDAL ${ }^{3}$, SEBASTIÁN CORNEJO ${ }^{4}$, DARÍO RAMÍREZ ${ }^{2}$, \\ DANILO MEDINAS ${ }^{5,6,7, a}$, GONZALO BUSTAMANTE ${ }^{2}$, \\ RENZO PASQUALI ${ }^{1}$, CLAUDIO HETZ ${ }^{5,6,7, b}$
}

\section{Creutzfeldt-Jakob disease. Report of one case}

Creutzfeldt-Jakob disease has a higher incidence in Chile than in other countries. The post mortem pathological characterization of brain tissue is necessary to reach a definitive diagnosis. We report a 73 years old man with a history compatible with of a rapidly progressive dementia, in which the first electroencephalographic study showed a pattern consistent with non-convulsive status epilepticus. Besides discarding this diagnosis, it was necessary to rule out other causes of rapidly progressive dementia such as Hashimoto encephalopathy. Finally, the sustained clinical deterioration with no response to anticonvulsants and corticosteroids, the imaging studies, a serial electroencephalographic monitoring study and the detection of 14-3-3 protein in cerebrospinal fluid were the keys to achieve the diagnosis of the disease.

(Rev Med Chile 2016; 144: 806-811)

Key words: Biomarkers; Creutzfeld-Jacob Syndrome; Electroencephalography; Status Epilepticus.

\section{L} a enfermedad de Creutzfeldt-Jakob (ECJ) es una enfermedad neurodegenerativa de curso fatal, producida por la acumulación de la proteína prion resistente a proteasas (Pr$\left.\mathrm{P}^{\mathrm{RES}}\right)^{1}$. La forma esporádica de la ECJ da cuenta de aproximadamente $85 \%$ de todos los casos. En Chile la incidencia es de 3,5 casos por millón de habitantes, siendo una enfermedad de notificación obligatoria ${ }^{2}$. El cuadro clínico de esta enfermedad se caracteriza por presentarse como una demencia rápidamente progresiva, acompañada comúnmente de mioclonías y ataxia. Se observan igualmente manifestaciones piramidales, extrapiramidales e incluso ceguera occipital. Posteriormente se observa una fase de mutismo acinético que se extiende hasta el fallecimiento del paciente ${ }^{3}$. Como herramientas de apoyo diagnóstico de la
ECJ se encuentran patrones electroencefalográficos característicos, así como también hallazgos típicos en la resonancia magnética (RM) cerebral y la presencia de la proteína 14-3-3 en líquido cefalorraquídeo $(\mathrm{LCR})^{4}$. El diagnóstico confirmatorio, sólo se logra mediante el estudio histopatológico post mortem.

\section{Caso clínico}

Se trata de paciente de sexo masculino de 73 años, proveniente de Santiago, sin hábitos alimenticios relevantes. Fue operado de cáncer de colon (etapa IIIC) en enero del año 2014, demás recibió tres ciclos de quimioterapia con oxaliplatino, última dosis en julio de 2014. El paciente acudió al 
Servicio de Urgencia del Hospital del Salvador en agosto de 2014, por cuadro de carácter progresivo de aproximadamente 7 meses de evolución que se había iniciado con insomnio de conciliación, no logrando dormir más de $3 \mathrm{~h}$ al día. Los meses siguientes los familiares lo notaron apático, con falta de iniciativa, dificultad para recordar nueva información y con inestabilidad de la marcha progresiva hasta impedir la deambulación. Comenzó a presentar episodios de desorientación e inatención fluctuantes, asociados a alucinaciones visuales complejas. Posteriormente era incapaz de responder atingentemente preguntas simples. En su evaluación, al ingreso, se objetiva un paciente vigil espontáneo, desorientado en el tiempo y espacio, inatento, con un lenguaje no fluente, no atingente y sin intención comunicativa. El paciente obedecía a órdenes simples, y era capaz de seguir con la mirada durante breves períodos de tiempo. Al examen motor destacó tono muscular aumentado, espástico, con reflejos osteotendíneos (ROT) aumentados vivos, simétricos y un reflejo plantar indiferente bilateral. El examen de pares craneanos, sensitivo, y de los signos meníngeos no evidenció alteraciones. El estudio de laboratorio general y de LCR resultó dentro de los parámetros normales esperados. La tomografía axial computarizada (TAC) de cerebro sin contraste resultó normal para la edad. Se planteó como diagnóstico sindromático una demencia rápidamente progresiva v/s un síndrome confusional.

$\mathrm{Al}$ inicio de la hospitalización presentó un episodio de crisis convulsiva tónico-clónica, la que fue manejada con benzodiacepinas con buena respuesta. El electroencefalograma (EEG) de vigilia evidenció un trazado anormal, marcadamente asimétrico, caracterizado a izquierda por la presencia continuos trenes de hasta 4 ondas theta de contornos agudos seguidos de una onda lenta y frecuentes punta con una máxima expresión en la región occipital (Figura 1A).

Ante la posibilidad de tratarse de un status epiléptico no convulsivo, se indicó un tratamiento con fenitoina en una dosis de carga. El paciente no presentó cambios clínicos, por lo que se agregó la administración de levetiracetam, nuevamente sin respuesta. Nuevos eletroencefalogramas mostraron evolución del trazado anterior hacia un registro caracterizado por la presencia de descargas periódicas lateralizadas a la izquierda, compuestas por complejos punta-onda o complejos doble o triple punta-onda con una frecuencia de $2 \mathrm{cps}$. La actividad a derecha se configuró esencialmente como ondas de morfología trifásica (Figura 1B).

Los días siguientes el paciente presentó mayor deterioro, encontrándose en un estado de sopor superficial, no emitía lenguaje y sólo localizaba estímulos dolorosos. Se realizó una RM de cerebro, la que mostró un incremento de la señal difusa a nivel neocortical, con mayor intensidad en el hemisferio izquierdo y en los ganglios basales (Figura 2A-C).

Dentro de los exámenes de laboratorio realizados en búsqueda de otras posibles causas de la demencia rápidamente progresiva, destacó la presencia de anticuerpos anti-tiro peroxidasa (anti-TPO) positivos. Ante este hallazgo se indicó manejo con corticoides (metilprednisolona $1 \mathrm{~g} /$ día por 5 días) sin obtener mejoría.

Un nuevo EEG fue realizado dos semanas después del anterior, el que evidenció un trazado caracterizado por la presencia generalizada de ondas de morfología trifásica, con una frecuencia aproximada de $1 \mathrm{cps}$, siempre de mayor expresión sobre el hemisferio izquierdo. Se observaron ocasionales depresiones de la amplitud y algunos episodios con aumento progresivo de la frecuencia de repetición de los complejos (Figura 1C).

Basado en la evolución clínica y en los hallazgos de la RM se planteó el diagnóstico de ECJ probable y se retiraron gradualmente los fármacos anticonvulsivantes. Se realizó un estudio bioquímico de la proteína 14-3-3 en LCR mediante Western blot, medido en distintas etapas del cuadro como se ha realizado anteriormente ${ }^{14}$, mostrando una mayor señal de ésta a medida que progresaba el deterioro clínico (Figura 2D).

El paciente evolucionó a una fase de mutismo acinético, agregando mioclonías de extremidades superiores. Luego de casi dos meses de hospitalización el paciente falleció. Consultada la familia respecto de la posibilidad de realizar una autopsia, deciden no llevar a cabo este procedimiento.

\section{Discusión}

En este artículo presentamos el caso de un paciente con un cuadro de demencia de curso subagudo en cuyo diagnóstico diferencial se consideró el status epiléptico no convulsivo y una posible encefalopatía respondedora a corticoides 
A A

B (1) (1)
Figura 1. Trazados de Electroencefalograma registrados a distintos tiempos del cuadro clínico. (A) Continuos trenes de actividad theta y puntas observados a izquierda con máxima expresión sobre regiones posteriores. (B) Descargas periódicas lateralizadas a izquierda a 2 cps que a derecha se configuran como ondas de morfología trifásica. (C) Descargas periódicas generalizadas de morfología trifásica a 1 $\mathrm{cps}$, ocasionales depresiones de amplitud y algunos episodios en curso de los cuales se aprecia un aumento progresivo de la frecuencia de repetición de los complejos. 

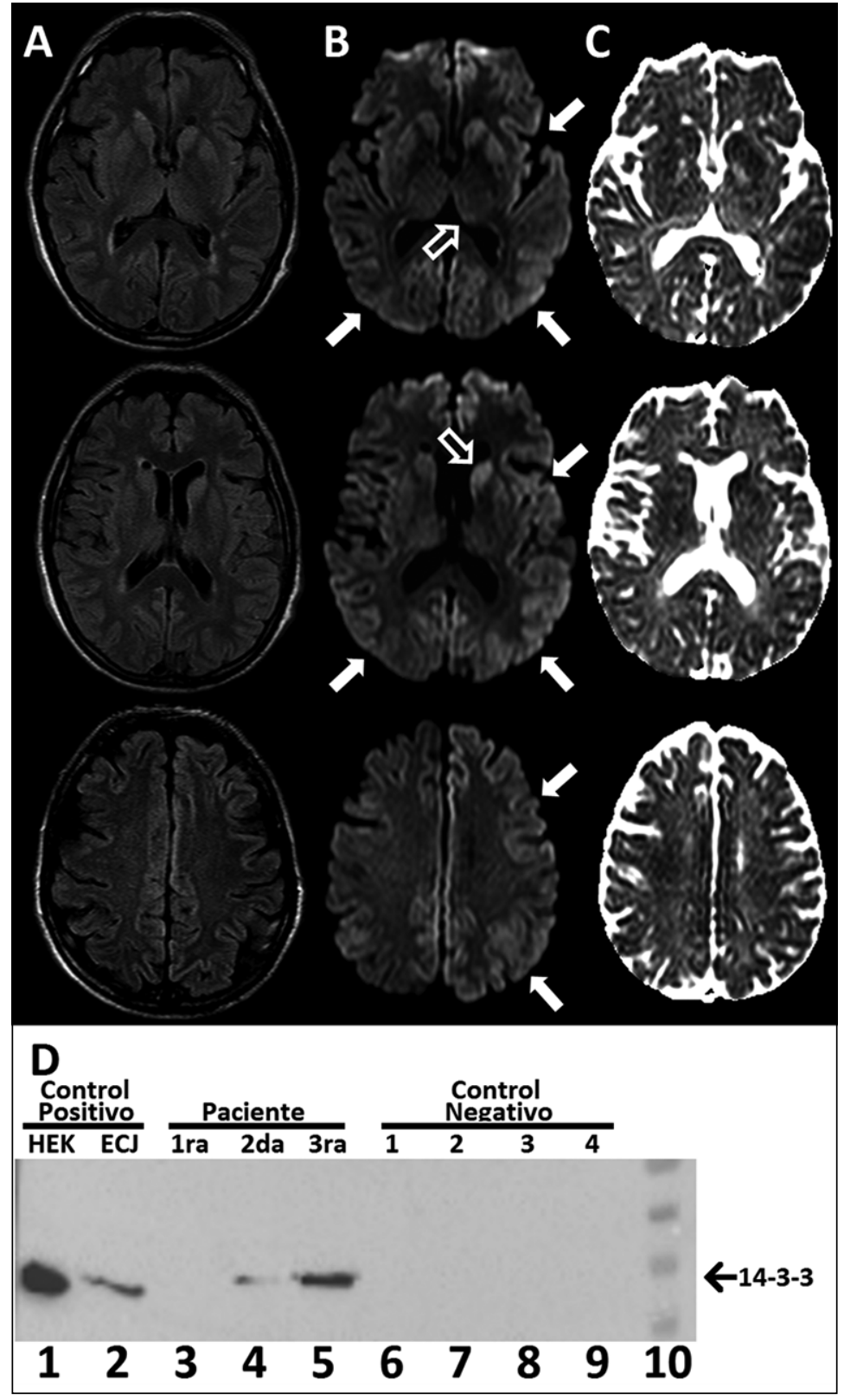

Figura 2. RM cerebral y detección de proteína 14-3-3. Imágenes transversales en FLAIR, difusión (DWI) y mapa ADC. Se evidencia un incremento de señal en FLAIR (A) con áreas de restricción de la difusión (B y C) a nivel neocortical en forma difusa en el hemisferio izquierdo y en territorios témporo-parietales a derecha (flechas sólidas en $\mathbf{B}$ ), asociado a alteraciones de similares características en los núcleos caudados (flecha transparente, segunda fila) y aspecto anterior de los putámenes en forma asimétrica, siendo mayor a izquierda. También se observó un pequeño foco hiperintenso en el pulvinar del tálamo izquierdo (flecha transparente, primera fila). No se evidenció compromiso de los hipocampos ni captaciones patológicas de contraste. (D) Detección de la proteína 14-3-3 a través de Western-blot. Se observa una acumulación progresiva de la proteína 14-3-3 del paciente con ECJ probable en muestras de LCR tomadas a distintos tiempos del cuadro (columnas 3 a 5). La 1ra muestra fue obtenida cercana al ingreso y la 3ra muestra en fase de mutismo acinético. Como control negativo se utilizaron muestras de LCR de donantes sanos (columnas 6-9). Como control positivo se utilizó una muestra de paciente con ECJ confirmada por biopsia que resultó ser positivo para 14-3-3 y lisado de células HEK (columnas 1-2). En la columna 10 se muestra el control de peso molecular. con anticuerpos anti-TPO positivos, antes de llegar al diagnóstico probable de ECJ.

En relación al status epiléptico no convulsivo, vale mencionar que la existencia de éste asociado a ECJ ya ha sido discutida en trabajos anteriores $^{5-9}$. Sin embargo, frente a la sospecha de un status epiléptico no convulsivo, siempre se deben considerar al menos 4 situaciones que obligan a descartar otras etiologías: 1) la existencia de un deterioro cognitivo agudo o sub-agudo, sin otra causa que lo explique; 2) una RM sin hallazgos significativos; 3) una falta de respuesta a tratamiento anticonvulsivante o a benzodiacepinas; y 4) un empeoramiento de una actividad de onda aguda en presencia de una mayor vigilia ${ }^{10}$.

El caso presentado constituye un desafío 
diagnóstico, sin embargo, fue adecuado realizar un tratamiento anticonvulsivante ante la sospecha de un status no convulsivo. El seguimiento electroencefalográfico fue esencial para lograr un mejor acercamiento etiológico del deterioro cognitivo subagudo puesto que el EEG puede cambiar en el curso del cuadro clínico y en algunos casos el patrón clásico aparece sólo tardíamente ${ }^{11}$. Por otro lado, hay que considerar también que el patrón clásico de ondas periódicas de morfología trifásica de frecuencia $1.5-2$ cps es idéntico al que se ve en muchas otras encefalopatías, por lo cual su especificidad se aplica sólo cuando existe una historia clínica sugerente.

Por su parte, la proteína 14-3-3 que es un polipéptido citosólico, que al estar presente en el LCR indicaría daño cerebral producto de muerte neuronal, tiene relevante valor como herramienta de apoyo diagnóstico. Su detección en el LCR tiene una sensibilidad de $94 \%$ y una especificidad de $84 \%$ en un contexto clínico adecuado ${ }^{12}$. Hay autores que prefieren la determinación de la proteína tau ya que contaría con una mayor especificidad ${ }^{13}$. Si bien la detección de la proteína 14-3-3 no genera un diagnóstico confirmatorio de la ECJ, se encuentra presente sólo en un bajo porcentaje de otros cuadros a plantear como diagnósticos diferenciales. En encefalopatía de Hashimoto se encuentra presente sólo en $12 \%$ de los casos, siendo incluso inferior en otras patologías autoinmunes. En el caso presentado destaca un notorio incremento en los niveles de proteína 14-3-3 al transcurrir el cuadro, siendo indudable su positividad en la etapa final, lo cual está en concordancia con lo descrito previamente ${ }^{14}$.

La RM es otra herramienta importante en el diagnóstico diferencial de una demencia rápidamente progresiva y tiene una alta sensibilidad y especificidad en el diagnóstico de ECJ, siendo las secuencias FLAIR y principalmente la difusión (DWI) las más importantes para el diagnóstico. En cerca de $90 \%$ de los casos se observan lesiones hiperintensas neocorticales, principalmente en regiones frontales, seguido de regiones parietales y temporales. En 70\% de los pacientes se observan lesiones estriatales, principalmente en el aspecto anterior de los putámenes y la cabeza de los núcleos caudados. Lesiones talámicas se observan aproximadamente en 35\% de los casos. Si bien hallazgos similares pueden ser encontrados en diferentes patologías tóxico-metabólicas, autoin- munes e infecciosas, la asociación de estas lesiones y su distribución asimétrica, visibles tanto en las secuencias FLAIR como en difusión, sugieren fuertemente el diagnóstico de $\mathrm{ECJ}^{15}$.

El antecedente de la cirugía coloproctológica reciente hace poco probable que este caso se tratase de una ECJ iatrogénica, ya que los menores tiempos de incubación reportados son los relacionados a material neuroquirúrgico contaminado con priones, ocurriendo el desarrollo de la enfermedad nunca en menos de un año ${ }^{16}$.

La biopsia es hasta ahora la única herramienta que permite confirmar el diagnóstico, sin embargo, este estudio no se realiza de rutina en Chile, siendo ésta la principal limitación de este trabajo. Sin embargo, tomando en conjunto los resultados del EEG, el análisis de la proteína 14-3-3 y la RM compatibles, se logra $98 \%$ de sensibilidad con una especificidad mayor a $70 \%{ }^{17}$. Aquí postulamos que el seguimiento, tanto electroencefalográfico como de biomarcadores, resulta relevante para lograr una buena sensibilidad diagnóstica. Dada la alta incidencia de ECJ en Chile, destacamos la urgente necesidad de contar con un centro de apoyo diagnóstico que permitan analizar e integrar la información clínica, imagenológica, neurofisiológica y neuropatológica de los pacientes con sospecha de ECJ. Es importante mencionar que estudios post mortem han demostrado que un porcentaje considerable de los casos reportados con la ECJ fallecieron por otras patologías tratables ${ }^{18}$. Además se requiere de nuevos exámenes que reemplacen o apoyen a los ya existentes y que permitan realizar el diagnóstico confirmatorio en pacientes vivos ${ }^{14,19,20}$ con el fin de identificar cuadros potencialmente reversibles y contribuir a una vigilancia epidemiológica de la enfermedad en nuestro país.

\section{Referencias}

1. Prusiner SB. Prions. Proc Natl Acad Sci U S A. 1998; 95 (23): 13363-83.

2. Brown P, Gálvez S, Goldfarb LG, Nieto A, Cartier L, Gibbs CJ Jr, et al. Familial Creutzfeldt-Jakob disease in Chile is associated with the codon 200 mutation of the PRNP amyloid precursor gene on chromosome 20. J Neurol Sci 1992; 112 (1-2): 65-7.

3. Henry C, Knight R. Clinical features of variant Creutzfeldt-Jakob disease. Rev Med Virol 2002; 12: 143-50.

4. Poser S, Mollenhauer B, Kraubeta A, Zerr I, Steinhoff BJ, Schroeter A, et al. How to improve the clinical diagnosis 
of Creutzfeldt-Jakob disease. Brain 1999; 122 (Pt 12): 2345-51.

5. Fernández-Torre JL, Solar DM, Astudillo A, Cereceda R, Acebes A, Calatayud MT. Creutzfeldt-Jakob disease and non-convulsive status epilepticus: a clinical and electroencephalographic follow-up study. Clin Neurophysiol 2004; 115 (2): 316-9.

6. Shapiro JM, Shujaat A, Wang J, Chen X. CreutzfeldtJakob disease presenting as refractory nonconvulsive status epilepticus. J Intensive Care Med 2004; 19 (6): 345-8.

7. Lapergue B, Demeret S, Denys V, Laplanche JL, Galanaud D, Verny M, et al. Sporadic Creutzfeldt-Jakob disease mimicking nonconvulsive status epilepticus. Neurology 2010; 74 (24): 1995-9.

8. van der Horn HJ, Egbers PH, Kuiper MA, Schuiling WJ. Diagnosing sporadic creutzfeldt-jakob disease in a patient with a suspected status epilepticus in the intensive care unit. Case Rep Neurol Med 2013; 2013: 630141.

9. Aiguabella M, Falip M, Veciana M, Bruna J, Palasí A, Corral L, et al. Refractory nonconvulsive status epilepticus in Creutzfeldt-Jakob disease. Epileptic Disord 2010; 12 (3): 239-42.

10. Freeman WD, Shuster E, Brazis P, Dickson D. Sporadic Creutzfeldt-Jakob disease mimicking nonconvulsive status epilepticus. Neurology 2011; 76 (12): 1111-2; author reply 1112 .

11. Wieser HG, Schindler K, Zumsteg D. EEG in Creutzfeldt-Jakob disease. Clin Neurophysiol 2006; 117 (5): 935-51.

12. Zerr I, Pocchiari M, Collins S, Brandel JP, de Pedro Cuesta J, Knight RS, et al. Analysis of EEG and CSF 143-3 proteins as aids to the diagnosis of Creutzfeldt-Jakob disease. Neurology 2000; 55 (6): 811-5.

13. Hamlin C, Puoti G, Berri S, Sting E, Harris C, Cohen $\mathrm{M}$, et al. A comparison of tau and 14-3-3 protein in the diagnosis of Creutzfeldt-Jakob disease. Neurology 2012; 79 (6): 547-52.

14. Torres M, Cartier L, Matamala JM, Hernández N, Woehlbier U, Hetz C. Altered prion protein expression pattern in CSF as a biomarker for Creutzfeldt-Jakob disease. Plos One 2012 Apr 7; 4.

15. Meissner B, Kallenberg K, Sánchez-Juan P, Collie D, Summers DM, Almonti S, et al. MRI lesion profiles in sporadic Creuzfeldt-Jakob disease. Neurology 2009; 72: 1994-2001.

16. Brown P, Brandel JP, Sato T, Nakamura Y, MacKenzie J, Will RG, et al. Iatrogenic Creutzfeldt-Jakob disease, final assessment. Emerg Infect Dis 2012; 18 (6): 901-7.

17. Tagliapietra M, Zanusso G, Fiorini M, Bonetto N, Zarantonello G, Zambon A, et al. Accuracy of diagnostic criteria for sporadic creutzfeldt-jakob disease among rapidly progressive dementia. J Alzheimers Dis 2013; 34 (1): 231-8.

18. Chitravas N, Jung RS, Kofskey DM, Blevins JE, Gambetti P, Leigh RJ, et al. Treatable neurological disorders misdiagnosed as Creutzfeldt-Jakob disease. Ann Neurol 2011; 70 (3): 437-44.

19. Moda F, Gambetti P, Notari S, Concha-Marambio L, Catania M, Park KW, et al. Prions in the urine of patients with variant Creutzfeldt-Jakob disease. N Engl J Med 2014; 371 (6): 530-9.

20. Orrú CD, Bongianni M, Tonoli G, Ferrari S, Hughson AG, Groveman BR, et al. A test for Creutzfeldt-Jakob disease using nasal brushings. N Engl J Med 2014; 371 (6): 519-29. 